\title{
Prediction of future rheumatoid arthritis in Chinese patients with early arthritis: An effective way of patient care.
}

\author{
Feifei Huang ${ }^{1 \#}$, Xianfeng Que ${ }^{1 \#}$, Hao Shen ${ }^{2 *}$ \\ ${ }^{1}$ Department of Orthopaedics, Affiliated Hospital of Nantong University, Nantong, Jiangsu, PR China \\ ${ }^{2}$ Department of Doppler Ultrasonic, Traditional Chinese Medicine of Nantong City, Nantong, Jiangsu, PR China \\ ${ }^{\#}$ Authors contributed to this work equally
}

\begin{abstract}
Objective: This was the first study to investigate the role of inflammatory biomarkers as predictors of future RA in Chinese patients with early onset of arthritis symptoms. The primary objective of this study was to check the involvement of inflammatory biomarkers as predictors of future RA in Chinese patients with early onset of arthritis symptoms.

Methods: Adult patients of either gender (aged between 20 to 65 years) who had mild sign and symptoms of arthritis (morning stiffness, mild swollen joint, mild pain in joints) were enrolled. The sign and symptoms of arthritis were measured in each enrolled patient. All the patients were followed up for 6-month intervals till clinical diagnosis of RA confirmed (max 3 year). Laboratory investigation was performed at every follow up visit.

Results: A total of 112 patients agreed to participate in our study, and willing to provide data as required by study, and completed the study. We found that the patients who had increased levels of rheumatoid factor of different immunoglobulin had high chance of developing RA. Similar trend was observed for antiperinuclear factor (APF) and antikeratin antibodies. The level of antinuclear antibodies was more among subjects who were diagnosed with RA than the subjects who don't have RA. Conclusion: Our study results suggested that the inflammatory biomarkers can be considered as predictors of future RA in Chinese patients with early onset of arthritis symptoms. Our study results support the hypothesis that biomarkers can be considered as one of the key predictors of future RA in Chinese patients with early onset of arthritis symptoms.
\end{abstract}

Keywords: Biomarkers, Arthritis, Rheumatoid arthritis, Chinese patients.

\section{Introduction}

Rheumatoid arthritis is one of most common cause of disability. Several lines of evidences showed that the early diagnosis of rheumatoid arthritis plays a vital role of prediction of outcome of diseases condition [1-6]. Prediction of future rheumatoid arthritis based on early sign and symptoms of early arthritis is one of the important achievements in current medical practice for improved patient care. For the optimal care and success of medical treatment, prediction of outcome of diseases condition after early sign and symptoms of arthritis with some accuracy plays a vital role in achieving success of medical intervention, and it is helpful for patients and consulting physician. Early prediction in outcome helps to improve healing potentials, and give chance to take decision about the future treatments and care intensity and to avoid ineffective diagnostic procedure. Moreover, the understanding of forthcoming consequences of patients' illness is the mainstay of the regaining process and improves patient care
[6-12]. In the emergency setting, clinical decision based on the patient's condition or illness has high impact on mortality and death among patients of critical illness [7-11].

Early changes in inflammatory biomarkers have been seen in patients with arthritis, particularly serum rheumatoid factors and autoantibodies [5-8]. Apart from the laboratory biomarker, there is few radiological diagnoses which helps in early detection of rheumatoid arthritis in patients with early arthritis or onset arthritis. The role of biomarkers has been established in other countries for early prediction of future RA in patients with the early onset of arthritis. However, the role of biomarkers has not been evaluated in Chinese patients with early onset of arthritis symptoms. This was the first nurse-led research designed to understand the association of biomarkers in predicting future RA in Chinese patients with early onset of arthritis symptoms for effective patient care. The main objective of this nurse-led research was to assess whether inflammatory biomarkers could be used as predictors of future RA in Chinese patients with early onset of arthritis symptoms. 
This nurse-led research may serve the basis for conducting large multi-centric, multi-country, clinical study which assesses the association of inflammatory biomarkers as predictors of future RA in Chinese patients with early onset of arthritis symptoms for effective patient care.

\section{Materials and Methods}

This pilot, single centre, nurse-led research, prospective study was designed to assess the involvement of inflammatory biomarkers as predictors of future RA in Chinese patients with early onset of arthritis symptoms. The assigned nurse enrolled the adult patients of either gender (aged between 20 to 65 years) who had mild sign and symptoms of arthritis (morning stiffness, mild swollen joint, mild pain in joints) at department of Department of Orthopaedics, Affiliated Hospital of Nantong University, China were included. The patients with severe sign and symptoms of arthritis or with the confirm diagnosis of RA or who were not willing to give written consent to participate in this study were excluded during screening phase of our study.

The assigned nurse has explained the study protocol to each patient who agreed to participate in this study. Written inform consent was taken from each patient or their relative to participate in this trial. This study and study protocol was review and approved by institutional ethics committee of Hospital of Nantong University, China. All the ethical principles which are laid down in Helsinki Declaration of 1964, as revised in 2013 were followed.

Blood samples $(5 \mathrm{ml})$ were obtained from each enrolled patient. Blood was collected into a tube containing potassium ethylenediaminetetraacetic acid (EDTA). Samples were analysed for inflammatory biomarkers such as serum rheumatoid factors, autoantibodies, human leukocyte antigenantigen D, immunoglobulin G-rheumatoid factors, immunoglobulin G-antikeratin antibodies, immunoglobulin G, antiperinuclear factor, CIP, CRP and ESR. Blood samples were drawn for inflammatory biomarkers analysis was stored at less than $80^{\circ} \mathrm{C}$. All data were collected subsequently. The sign and symptoms of arthritis were measured in each enrolled patient. All the patients were followed up for 6-month intervals till clinical diagnosis of RA confirmed (max 3 year). Laboratory investigation was performed at every follow up visit.

Since this study was a pilot study, no formal sample size calculation was performed. However, we have planned to include at least 100 Chinese patients with mild sign and symptoms of arthritis (morning stiffness, mild swollen joint, mild pain in joints) in this study. Correlations of inflammatory biomarkers with arthritis in Chinese patients were done using Pearson's correlation/regression models. Relationship with biomarkers and RA in Chinese patients with early arthritis was analysed using Pearson correlation coefficient. Numerical variable was presented as mean (standard deviation). Qualitative outcome was presented as absolute number (percentage) of individuals in each category. Statistical analysis was performed using version 6.2 of Graph Pad Prism.

\section{Results}

We identified a total of the 132 medical records of patients during January 2013 to December 2016 who were visited in our hospital after having sign and symptoms of arthritis at the time of their consultation. Of these, a total of 112 patients agreed to participate in our study, and willing to provide data as required by study, and completed the study. Majority of identified patients were men (male: 80 , female: 32 ) with mean (SD) age of 38 (2.1) years. Demographic and clinical characteristic of patients who diagnosed with RA are presented in Table 1.

Table 1. Demographic and clinical characteristic of patients who diagnosed with $R A$.

\begin{tabular}{ll}
\hline Variable & $\begin{array}{l}\mathbf{N}=112 \\
\mathbf{n}(\%)\end{array}$ \\
\hline Age, year, Mean (SD) & $38(2.1)$ \\
\hline Male/female, $\mathrm{n}$ & $80 / 32$ \\
\hline BMl, kg/m², Mean (SD) & $25.14(1.3)$ \\
\hline Joints with symptoms of arthritis, Mean (SD) & $4.1(2.3)$ \\
\hline Type 2 diabetes mellitus, \% & $09 \%$ \\
\hline Hypertension, \% & $05 \%$ \\
\hline Atherosclerosis, \% & $04 \%$ \\
\hline Dyslipidemia, \% & $06 \%$ \\
\hline
\end{tabular}

Values are presented as Mean (SD) or as absolute number (\%); $\mathrm{N}=$ Total number of subjects; $n=$ number of subjects in each category.

Among inflammatory biomarkers, the levels of ESR levels were comparable in among subject with RA and Non-RA. The levels of ESR were numerically greater among subject with RA. Similar trend was observed for CRP levels. The levels of CRP were numerically greater among subject with RA. We found that the patients who had increased levels of rheumatoid factor of different immunoglobulin had high chance of developing RA. Similar trend was observed for antiperinuclear factor (APF). When antikeratin antibodies (AKA) associated with Ig were compared, we found greater levels of AKA associated with $\operatorname{IgA}$, except $\operatorname{IgM}$ and IgG. The level of antikeratin antibodies related with $\operatorname{IgA}$ was greater among the subjects with RA as compared to subjects with no RA. The level of antinuclear antibodies was more among subjects who were diagnosed with RA than the subjects who don't have RA. Circulating immune complexes associated with Ig were compared; we found that there was no statistical significant difference in immune complexes associated with IgM, IgG and IgA. We also found that there was no significant difference in IgG levels (IgG1, IgG 2, IgG3, IgG4 and IgG5) among the patients who were diagnosed with RA as compared to patients not diagnosed with RA. The proportion of patients with human leukocyte antigen-antigen D was significantly higher among the patients who were diagnosed with RA as compared to subjects with no RA (Table 2). The variable for which 
difference was noted as statistically significant, were also found correlated with RA (Table 3 ).

Table 2. Change in levels of difference biomarkers in patients who were diagnosed with $R A$ and non-RA patients.

\begin{tabular}{|c|c|c|c|}
\hline Final disposition of RA & $\begin{array}{l}\text { Pts with RA } \\
(n=50) \\
\text { (Median) }\end{array}$ & $\begin{array}{l}\text { Pts with not } \\
\text { RA } \\
\text { (n=62) } \\
\text { (Median) }\end{array}$ & $P$ values \\
\hline $\begin{array}{l}\text { Erythrocyte sedimentation } \\
\text { rate }\end{array}$ & 20.1 & 20 & $\begin{array}{l}\text { Not significant } \\
\text { (NS) }\end{array}$ \\
\hline C-reactive protein & 17 & 12 & $\begin{array}{l}\text { Not significant } \\
\text { (NS) }\end{array}$ \\
\hline Rheumatoid factor-IgM & 0.28 & 0.18 & $<0.005$ \\
\hline Rheumatoid factor-lgG & 0.18 & 0.12 & $<0.005$ \\
\hline Rheumatoid factor-IgA & 0.32 & 0.12 & $<0.005$ \\
\hline Antiperinuclear factor & $2 / 80$ & 0 & $<0.005$ \\
\hline Antikeratin antibodies-IgM & 0.17 & 0.16 & $>0.005$ \\
\hline Antikeratin antibodies-IgG & 0.22 & 0.21 & $>0.005$ \\
\hline Antikeratin antibodies-lgA & 0.31 & 0.2 & $<0.005$ \\
\hline Antinuclear antibodies & $3 / 23$ & $1 / 23$ & $<0.005$ \\
\hline $\begin{array}{l}\text { Circulating } \\
\text { complexes-IgM }\end{array}$ & 0.18 & 0.11 & $>0.005$ \\
\hline $\begin{array}{l}\text { Circulating } \\
\text { complexes-lgG }\end{array}$ & 0.28 & 0.12 & $>0.005$ \\
\hline $\begin{array}{l}\text { Circulating immune } \\
\text { complexes-lgA }\end{array}$ & 0.22 & 0.12 & $>0.005$ \\
\hline IgG one & 0.28 & 0.29 & $>0.005$ \\
\hline $\lg G$ two & 0.48 & 0.40 & $>0.005$ \\
\hline IgG three & 0.52 & 0.53 & $>0.005$ \\
\hline $\lg G$ four & 0.42 & 0.43 & $>0.005$ \\
\hline $\begin{array}{l}\text { Human leukocyte antigen- } \\
\text { antigen D, \% }\end{array}$ & $67 \%$ & $32 \%$ & $<0.005$ \\
\hline $\begin{array}{l}\text { Values are presented as } \mathrm{Me} \\
\text { number of subjects; } n=\text { number }\end{array}$ & $\begin{array}{l}\text { an }(\mathrm{SD}) \text { or } \\
\text { of subjects in }\end{array}$ & $\begin{array}{l}\text { Is absolute numbe } \\
\text { each category }\end{array}$ & er $(\%) ; \quad N=$ Total \\
\hline
\end{tabular}

\section{Discussion}

This was the first nurse-led research designed to understand the association of biomarkers in predicting future RA in Chinese patients with early onset of arthritis symptoms for effective patient care. Early prediction in outcome helps to improve healing potentials, and give chance to take decision about the future treatments and care intensity and to avoid ineffective diagnostic procedure. Moreover, the understanding of forthcoming consequences of patients' illness is the mainstay of the regaining process.

In our study, the levels of ESR levels were comparable in among subject with RA and Non-RA. The levels of ESR were numerically greater among subject with RA. Similar trend was observed for CRP levels. We also found that the patients who had increased levels of rheumatoid factor of different immunoglobulin had high chance of developing RA. Similar trend was observed for antiperinuclear factor (APF). When antikeratin antibodies (AKA) associated with Ig were compared, we found greater levels of AKA associated with $\operatorname{IgA}$, except IgM and IgG. The level of antinuclear antibodies was more among subjects who were diagnosed with RA than the subjects who don't have RA. The proportion of patients with human leukocyte antigen-antigen D was significantly higher among the patients who were diagnosed with RA as compared to patients not diagnosed with RA.

Table 3. Relationship with biomarkers and RA in Chinese patients with early arthritis.

\begin{tabular}{lll}
\hline \multirow{2}{*}{ Key biomarkers } & $\begin{array}{l}\text { RA } \\
(\mathbf{N}=50)\end{array}$ & \\
\cline { 2 - 3 } & Beta-Coefficients & P value $^{*}$ \\
\hline Rheumatoid factor-lgM & -0.53 & $<0.005$ \\
\hline Rheumatoid factor-IgG & -0.58 & $<0.005$ \\
\hline Rheumatoid factor-IgA & -0.47 & $<0.005$ \\
\hline Antiperinuclear factor & -0.62 & $<0.005$ \\
\hline Antikeratin antibodies-lgA & -0.43 & $<0.005$ \\
\hline Antinuclear antibodies & -0.49 & $<0.005$ \\
\hline $\begin{array}{l}\text { Human leukocyte antigen-antigen } \\
\text { D }\end{array}$ & -0.51 & $<0.005$ \\
\hline
\end{tabular}

$\mathrm{N}=$ Total number of subjects; $\mathrm{P}$ values were calculated using Pearson correlation coefficient.

Our study is the first nurse-led research study suggests the role of inflammatory biomarkers as predictors of future RA in Chinese patients with early onset of arthritis symptoms. Since the study was designed as pilot study and conducted at single study centre in China (limitation of study). Therefore, the present findings cannot be generalized to the overall Chinese population. Based on the study results, we suggest for conducting large multi-centric randomized clinical study in future to generalize our findings.

\section{Conclusion}

These results of this nurse-led research suggested that the inflammatory biomarkers can be considered as predictors of future RA in Chinese patients with early onset of arthritis symptoms for effective patient care. Our study results support the hypothesis that biomarkers can be considered as one of the key predictors of future RA in Chinese patients with early onset of arthritis symptoms.

\section{Acknowledgement}

All authors would like to thank all the subjects for their participation. 


\section{Conflict of Interest}

The authors declare that there is no conflict of interest.

\section{References}

1. Irvine S, Munro R, Porter D. Early referral, diagnosis, and treatment of rheumatoid arthritis: evidence for changing medical practice. Ann Rheum Dis 1999; 58: 510-513.

2. Van de putte LBA, Van Gestel AM, Van Riel PLCM. Early treatment in rheumatoid arthritis: rationale, evidence and implications. Ann Rheum Dis 1998; 57: 511-512.

3. Mikuls TR, O'Dell J. The changing face of rheumatoid arthritis therapy: results of serial surveys. Arthritis Rheum 2000; 43: 464-465.

4. Walker DJ, Pound JD, Griffiths ID, Powell RJ. Rheumatoid factor tests in the diagnosis and prediction of rheumatoid arthritis. Ann Rheum Dis 1986; 45: 684-690.

5. Wolfe F, Cathey MA, Roberts FK. The latex test revisited: rheumatoid factor testing in 8287 rheumatic disease patients. Arthritis Rheum 1991; 34: 951-960.

6. Arnett FC, Edworthy SM, Bloch DA, McShane DJ, Fries $\mathrm{JF}$, Cooper. The American rheumatism association revised criteria for the classification of rheumatoid arthritis. Arthritis Rheum 1988; 31: 315-324.

7. Bla $\beta$ S, Engel JM, Burmester GR. Review: the immunologic homunculus in rheumatoid arthritis. Arthritis Rheum 1999; 42: 2499-2506.
8. Saraux A, Berthelot JM, Le Henaff C, Chales G, Thorel JB, Hoang S. Classification and diagnostic value of the 1987 ACR criteria for rheumatoid arthritis (RA) in early arthritis (EA). Arthritis Rheum 2000; 43: 154.

9. Saraux A, Bendaoud B, Dueymes M, LeGoff P, Youinou P. Functional affinity of IgM rheumatoid factor in rheumatoid arthritis. Ann Rheum Dis 1997; 56: 126-129.

10. Youinou P, Le Goff P, Dumay A, Lelong A, Fauquert P, Jouquan J. The antiperinuclear factor. I. Clinical and serological associations. Clin Exp Rheumatol 1990; 8: 1-6.

11. Meyer O, Tauxe F, Fabregas D, Gabay C, Goycochea M, Haim T, Elias A, Kahn MF. Anti-RA 33 antinuclear antibody in rheumatoid arthritis and mixed connective tissue disease: comparison with antikeratin and antiperinuclear antibodies. Clin Exp Rheumatol 1993; 11: 473-478.

12. Laemmli UK. Cleavage of structural proteins during the assembly of the head of bacteriophage T4. Nature 1979; 227: 680-685.

\section{Correspondence to:}

Hao Shen

Department of Doppler Ultrasonic

Traditional Chinese Medicine of Nantong City

PR China 